einem genetiv Lolli, wie er $\%$. $b$. auch auf den vasen CII. XII, $5686,489 \mathrm{~b}$ - e begegnet, der nominativ als Lollius oder Lollus angesetzt werden kann. Wenn nun unser interpolator im alten Sugamberngebiet lebte und ihm auch dort eine inschrift bekannt wurde, auf welcher der name Lollius oder ähnlich stand - vielleicht als einziger lesbarer rest -, so konnte ihn dies wohl anregen, darauf sein Lollusmärchen aufzubauen. Schade, daß wir die ausgabe des Nomesseius nicht mehr besitzen! Nach der einen uns bekannt gewordenen probe seines verfahrens dürften wir erwarten, in seinem text auch noch andere amüsante fallstricke für allzu gutgläubige leser zu finden.

GIESSEN, 18. oktober 1917.

KARI, HELM.

\title{
ZUM MD. GEDICHT VON DHR JUDITH.
}

Das in der Mergentheim-Stuttgarter hs. HB 13 poet. germ. 11 überlieferte mhd. gedicht von der Judith pflegt als ältestes werk der deutschordensdichtung gezählt zu werden, so von Hering in seiner dissertation ${ }^{1}$ ), so auch von mir in meinem zusammenfassenden überblick über die ordensliteratur ${ }^{2}$ ). Aber die sachlage ist nicht so einfach, wie es scheint: die zugehörigkeit zur deutschordensdichtung wie auch die entstehungszeit - diese trotz der in der hs. gemachten bestimmten angabe - verlangen weitere erörterung, zumal beide in einem gewissen schwer verständlichen gegensatz stehen. Ja dieser gegensatz. ist so stark, $\mathrm{da} \beta$ sich beinahe sagen läßt: wenn die im gedicht genannte entstehungszeit 1254 richtig ist, kann das werk schwerlich dem deutschordenskreis zugehören - und umgekehrt, wenn es in diesem kreis entstand, kann es schwerlich aus dem jahre 1254 stammen.

Bei der besprechung der literarhistorischen stellung des gedichtes legt Hering in erster linie gewicht anf die iiber-

1) Max Hering, Untersuchungen uber Judith, ein mitteldeutschics gedicht des 13. jalhrhunderts. Diss. Halle (1907), s. 12.

2) Die literatur des dentschen ordens im mittelalter, Zs. f. d. A. unterricht 30,294 f. 
lieferung. Die einzige handschrift, in welcher das gedicht erhalten ist, wurde jedenfalls im kreise des deutschen ordens zusammengestellt und enthält im übrigen nur werke, die mit sicherheit dem deutschen orden auch ihre entstehıng verdanken '). I)as ist gewiß ein schwerwiegendes indicium, aber doch nicht vollbeweisend, da der orden bekanntlich auch werken anderer herkunft seine pflege zukommen ließ, wenn ihr inhalt für ihn interesse bot ${ }^{2}$ ), was bei einem Judithgedicht selbstrerständlich der fall war.

Aus der mundart des werkes läßt sich für unsere frage nichts schließen. Seine sprache zeigt in hohem grade thüringische eigentümlichkeiten ${ }^{3}$ ). 1)a Thüringen die älteste ballei des ordens in Deutschland war und gerade im 13. jh. das thüringische element im orden eine große rolle spielte, würde die mundart des gedichtes gut zu einer entsteliung des gedichtes im kreise des ordens passen, aber beweisen läßt sich solche entstehung damit nicht. Daß der verfasser etwa selbst schon aus seiner thüringischen heimat ins ordensland gezogen sei, zeigt sich in dem lautlichen gewand seiner sprache in keiner weise. Wir dürften das freilich, auch wenn diese wanderung stattgefunden hätte, nicht erwarten; denn der dichter war nach seiner eigenen angabe noch junc (v. 677; vgl. Hering s. 11), es kann also noch kein längerer aufenthalt in der fremde angesetzt werden, der seine mundart hätte wandeln können. Ist übrigens das gedicht wirklich bereits um die mitte des 13. jh.'s geschrieben, so würde es in eine zeit fallen, in welcher im ordensland eine gemeinsprache, der er sich hätte anschließen können, auch in ihren anfängen noch gewiß nicht existierte.

Wichtiger ist der wortschat: ${ }^{4}$ ). Er zeigt einige verwandtschaft mit dem wortschatz anderer ordensdichtungen, und wenn auch nicht alles, was Hering zusammengestellt hat, in diesem sinne verwertet werden darf, so bleibt doch noch ein selrr beachtenswertes material übrig. Nur beachte 'man

1) Vgl. auch meine Maccabäerausgabe, s. 77, wo ich auch zuerst betonte, daß für die Judith diese herkunft nicht so selbstverständlich ist.

2) Vgl. Zs. f. d. d. unterr. 30, 436 .

3) S. Hering s. $29 \mathrm{ff}$.

4) Zusammenstellungen bei Hering s. $02 \mathrm{ff}$. 
dann auch, daß jene anderen ordensdichtungen nicht der mitte des 13. jh.'s angehören, sondern sämtliche nicht unerheblich, zum teil sogar sehr beträchtlich jünger sind. Der wortschatz ist also zunächst geeignet, einigen zweifel an der bisherigen zeitlichen ansetzung des gedichts zn erwecken. .

Die wenigen persönlichen bemerkungen des dichters ergeben für unsere frage gar nichts. Die von ihm mehrfach angewendete anrede an seinen freund vrunt und bruder min in gote u. ä. (s. Hering s. 13) ist natïrlich nicht notwendigerweise auf einen deutschordensbruder zu deuten; sie ist ebenso gut am platze, wenn er irgend einem mönchsorden angehörte, ja man kann fragen, ob nicht auch weltgeistliche untereinander sich als vrunt und bruder in gote bezeichnen kounten.

In diesem zusammenhang miissen die verse $640 \mathrm{ff}$. betrachtet werden, wo es heißt:

640 got der scherfe mir den sin
vnd hute min in siner wer,
daz mich da ron icht iage der ber,
davon man an dem donrestage
liset in hern Davides sage
545 vnd gote so claget: horet,
den wingarten zustoret
uz dem walde hat der ber.

Hering (s. 12) hat erkannt, daß eine anspielung auf Psalm 79, 14 vorliegt, es ist ihm aber offenbar entgangen, $d a ß$ in den worten an dem donrestage ein deutlicher hinweis auf das breviergebet enthalten ist. Die verteilung der psalmen auf die wochentage ist alt ${ }^{1}$ ). Sie findet sich als feste regel in der benediktinischen liturgie, die indessen ihrerseits offenbar auf der römischen nach der tradition durch den heiligen Gregor festgesetzten liturgie fuBt ${ }^{2}$ ). Nach einer freundlichen mitteilung des herrn paters Anselm Manser O. S. B. zu Beuron beten auch heute die Benediktiner den Psalm 79 am donnerstag und zwar in der zweiten nocturn an erster stelle. Aber der gebrauch beschränkte sich nicht auf diesen orden, sondern ging frïh darüber hinaus; der psalm stand nach demselben gewährsmann

1) Vgl. P. Suitbert Baumer, (Heschichte des breviers, versuch einer quellenmaßigen darstellung der entwicklung des altkirchlichen und des römischen officiums (Freiburg. 1. Br. 1895), s. $246 \mathrm{fr}$.

2) A. A. 0. A. $203 \mathrm{ff} .213 \mathrm{ff}$, 
auch schon im mittelalter in der donnerstags-matutin des breviers der weltgeistlichen an letzter stelle. Wie es sich in anderen orden verhält, entrieht sich meiner kenntnis. Der deutsche ritterorden kannte selbstverständlich ebenfalls das stundengebet. Artikel 8 der regel ${ }^{1}$ ) bestimmt darüber: die brudere phaffen unde leigen sulen gemeinliche lumen tages unde nahtes zu gotes dieneste uncle zu ir geziten, die phaffen durch daz si singen nach den brevieren unde buchen, die nach dem orden geschriben sint. Und daß auch in ihrem brevier die psalmen enthalten sind, wird - so selbstverständlich es ist obendrein bestätigt, wenn es in demselben artikel später heißt: swelcher (der leigen brudere) von im selber oder mit urlobe des obersten mit den phaffen die tagezit oder die gezit von unser vrowen an den salmen unde an anderen dingen die zu dem ambehte gehorent der phaffen, sprechen wollent... Welche anordnung der psalmen nun aber die breviere, dic nach dem orden geschriben sint, zeigten, das wissen wir nicht. Es ist aber nach der geschichte des breviers an der möglichkeit vorläufig nicht zu zweifeln, daß auch im ordensbrevier Psalm 79 auf den donnerstag fiel, die verse bilden mithin wenigstens kein hindernis, das werk dem ordenskreis zuzuweisen.

Für die zugehörigkeit zur ordensdichtung spricht nun außer dem wortschatz noch besonders ein umstand: die übertragung biblischer bücher ist im orden als eine specialität gepflegt worden ${ }^{2}$ ) und besonders die übertragung alttestamentlicher bücher, deren inhalt sich mit kämpfen für den glauben im weitesten sinne befaßt: Daniel, Esther, Maccabäer. Ferner ist für die ordensdichtung charakteristisch die unterbrechung des textes solcher erzählender werke durch mystisch-allegorische auslegungen. Auch dies ist in der Judith schon: vorhanden und wiegt meines erachtens schwer. Indessen ist die so gerichtete literarische betätigung im orden auf jahrzehnte beschränkt, die später liegen. 'Auch wenn wir absehen von den jahren, in welchen Luder von Braunschwèig in dieser richtung wirkte und auch früheres mit in betracht ziehen, so kommen

1) Die statuten des deutschen ordens, hrsg. von M. Perlbach (Halle 1890), s. 34.

2) Vgl. A. Hübner, Daniel, eine deutschordensdichtung (Berlin 1911), s. $85 \mathrm{ff}$; Helın, Zs. f̣. d. d. unterr. 30, 295. 
wir doch nur mit der Esther in das ende des 13. oder den anfang des 14. jh.'s. Auch von hier aus erheben sich also zweifel an der entstehung der Judith im jahre 1254.

Ist aber ein solcher zweifel an der bestimmten angabe im gedicht selbst überhaupt erlaubt? Die datierung geschieht dort v. $2766 \mathrm{ff}$. mit folgenden worten:

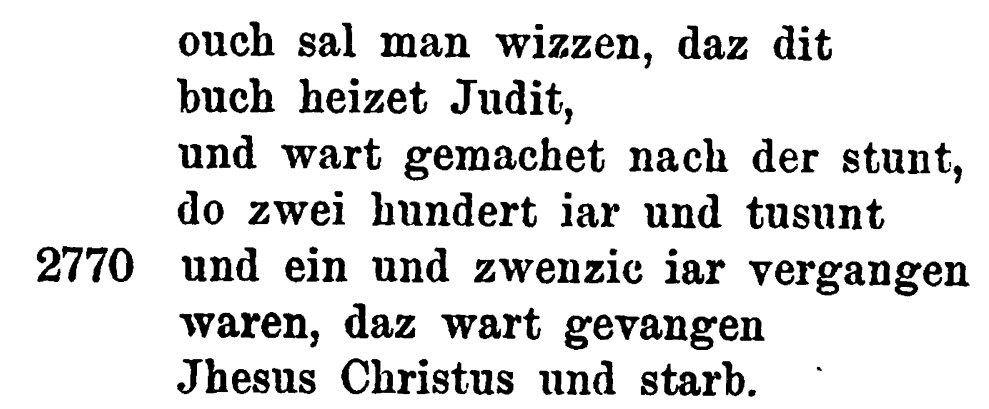

Das jahr der kreuzigung $33+1221$ ergibt 1254 . Eine fälschung ist natürlich ausgeschlossen. Aber ein schreibfehler wäre möglich, da die handschrift ja eine ältere niederschrift copieren mußte. Wahrscheinlich wird aber ein solcher schreibfehler nur, wenn er sich aus paläographischen einzelheiten leicht ergeben kann. In unserem fall liegt diese möglichkeit nun sehr nahe. Ich nehme an, daß in v. 2770 statt zwenzic, wie die Mergentheimer handschrift schreibt, ursprünglich zibenzic stand.

Zur weiteren begründung meiner vermutung nnd der angenommenen falschen abschrift beachte man folgendes.

1. Die schreibung $z$ für anlautendes $s$ ist in handschriften des 14. und 15. jh.'s vielfach $\mathrm{zu}$ finden, besonders im mitteldeutschen. Fs dürfte überflüssig sein, material, das jedermann leicht finden kann, hier zusammenzustellen. Besonders leicht kann sich $z$ aber wohl einstellen in einem falle wie dem unseren, wo es in zusammenhang mit dem vorhergehenden und eine sandhierscheinung $(n d+s>n d+z)$ sein kann. Ganz ebenso schreibt die hs. W des Wiener Oswald v. 569 zwe und zebeczig, doch ist dort $z$ für $s$ iiberhaupt sehr häufig; vgl. G. Baesecke Der Wiener Oswald, s. XIV.

Auch der umgekehrte fall, da $B$ im anlaut $s$ für $z$ geschrieben wird, ist bekannt, und auch dieser wenn auch seltenere schreibgebrauch könnte an unserer stelle mitgewirkt haben. Er scheint namentlich in der anlantverbindung mit $w$ aufz.lltreten, vgl. die belege bei Weinhold, Mhd. gramm. $\$ 205$, ferwer 
in der Rennerhs. B die formen swentzig. swolffe (Ehrismann, Der Renner 4, 33). Der schreiber der Mergentheimer hs. könnte also auch ein vorliegendes sibenzic, dessen $i b$ er als 10 las ( S. unten) als swenzic gelesen und in zwenzic gebessert haben.

2. Die schriftbilder $i b$ und $w$ können sich in den handschriften sehr nahe stehn, namentlich wenn ein schreiber beim $v$ den zweiten strich etwas höher macht als den letzten und zwischen dem ersten und zweiten strich die verbindung nur unvollkommen ausfïhrt. So ist es $z$. b. liäufig in der Danziger hs. der Heslerschen apokalypse; vgl. das facsimile in den deutschen texten des mittelalters, bd. XIV, tafel I. Dort könnte in spalte 2, z. 6 v. u. das wort zwiualdes leicht als zibiualdes gelesen werden. Auch die Königsberger hs. $891 \mathrm{~b}$ hat manche derartige $w$. Wenn solche verwechslung möglich ist, kann aber natürlich ungekehrt statt $i b$ auch einmal $w$ gelesen werden, und diese annahme ist nicht lediglich theorie; ich bin in der lage, ein einwandfreies beispiel für solche verlesung anzuführen. Die Olmützer hs. des Wiener Oswalds hat in v. 49 die zahl 72, die dort sogar an einer stelle steht, wo sie formelhaft ist 1 ) und deshalb ganz besonders fest sein sollte, durch 22 ersetzt; ihr schreiber hat also ganz zweifellos ein in seiner vorlage stehendes zibenzic für zwenzic verlesen.

Nehmen wir für die Judithstelle in der Mergentheimer hs. denselben vorgang an, so erhalten wir eine ursprüngliche zahlenangabe 1271 statt 1221 und kommen auf $33+1271=1304$ als jahr der abfassung der Judith, womit die schwierigkeiten, die sich bei der früheren datierung aus dem wortschatz und dem ganzen charakter des werkes ergaben, wegfallen. Die annahme, daß das werk zur deutschordensdichtung gehört - allerdings nun nicht mehr als ihr ältestes erzeugnis -, kann durch die beseitigung dieses anstoßes an sicherheit nur gewinnen.

1) Es handelt sich um die 72 völker, die Tragemunt kennt.

GIESSEN, den 14. juli 1917.

KARL HELM. 